\title{
Hepa1-6-FLuc cell line with the stable expression of firefly luciferase retains its primary properties with promising bioluminescence imaging ability
}

\author{
YASHA LI ${ }^{1-3}$, MENGNAN LIU ${ }^{1,2}$, JIEJIE CUI ${ }^{1-3}, \mathrm{KE} \mathrm{YANG}^{1,3}, \mathrm{LI} \mathrm{ZHAO}^{1,3}$, \\ MENGJIA GONG ${ }^{1,3}$, YI WANG ${ }^{2}$, YUN HE ${ }^{2}$, TONGCHUAN HE ${ }^{1-3}$ and YANG BI ${ }^{1-3}$
}

\author{
${ }^{1}$ Stem Cell Biology and Therapy Laboratory, Ministry of Education Key Laboratory of Child Development and Disorders; \\ ${ }^{2}$ Department of Pediatric Surgery; ${ }^{3}$ Key Laboratory of Pediatrics in Chongqing, International Science and \\ Technology Cooperation Base of Child Development and Critical Disorders, The Children's Hospital of \\ Chongqing Medical University, Chongqing 400014, P.R. China
}

Received October 31, 2016; Accepted January 1, 2018

DOI: $10.3892 / \mathrm{ol} .2018 .8132$

\begin{abstract}
Reliable animal models are required for the in vivo study of the molecular mechanisms and effects of chemotherapeutic drugs in hepatocarcinoma. In vivo tracing techniques based on firefly luciferase (FLuc) may optimize the non-invasive monitoring of experimental animals. The present study established a murine Hepa1-6-FLuc cell line that stably expressed a retrovirus-delivered FLuc protein gene. The cell morphology, proliferation, migration and invasion ability of Hepa1-6-FLuc cells were the same as that of the Hepa1-6 cells, and thus is suitable to replace Hepa1-6 cells in the construction of hepatocarcinoma animal models. No differences in subcutaneous tumor mass and its pathomorphology from implanted Hepa1-6-FLuc cells were observed compared with Hepa1-6 control tumors. Bioluminescence imaging indicated that the Luc signal of the Hepa1-6-FLuc cells was consistently strengthened with increases in tumor mass; however, the Luc signal of Hepa1-6-AdFLuc became weaker and eventually disappeared during tumor development. Therefore, compared with the transient expression by adenovirus, stable expression of the FLuc gene in Hepa1-6 cells may better reflect cell proliferation and survival in vivo, and provide a reliable source for the establishment of hepatocarcinoma models.
\end{abstract}

Correspondence to: Dr Yang Bi, Stem Cell Biology and Therapy Laboratory, Ministry of Education Key Laboratory of Child Development and Disorders, The Children's Hospital of Chongqing Medical University, Building 7, Room 905, 136 Zhongshan Er Road, Chongqing 400014, P.R. China

E-mail: yang_bi@hospital.cqmu.edu.cn

Key words: firefly luciferase, bioluminescence imaging, hepatocellular carcinoma cells

\section{Introduction}

Primary hepatocellular carcinoma (HCC) is a human malignancy with a high incidence, mortality and recurrence rate, worldwide, seriously threatening human health $(1,2)$. China is one of the areas of high HCC incidence, $>45 \%$ of the worldwide cases of HCC were estimated to occur in China in the last decade. In addition, HCC is the second leading cause of malignant tumor mortality in males in China, after gastric carcinoma $(3,4)$. For these reasons, research regarding liver carcinoma treatment is essential.

The orthotopic transplantation tumor model of HCC is an ideal model for studying the mechanisms of metastasis and tumor immunity, and for the development of anti-tumor drugs and novel therapeutic methods $(5,6)$. Techniques of cell tracking in vivo may permit the noninvasive monitoring of experimental animals, which is of great significance for the dynamic study of tumor diseases. Commonly used in vivo tracing techniques include radionuclide imaging, magnetic resonance imaging and optical imaging $(7,8)$. Among these methods, in vivo optical imaging technology with bioluminescence (bioluminescence image, BLI) has the advantages of high sensitivity, accurate quantification with minimal trauma, simple operation and the capacity for direct observation. At present, it is utilized extensively in preclinical cancer studies, including stem cell tracking, progression of tumor metastasis or the kinetics of tumor growth, to assess the effectiveness of antineoplastic agents in a tumor xenograft mouse model (9-11).

The murine hepatoma Hepa1-6 cell line, originating from a BW7756 mouse hepatoma in a C57/L mouse, is commonly used to establish hepatocarcinogenesis mouse models due to its high malignancy and low immunogenicity (12). In the present study, the potential application of the Hepa1-6 cell line transfected with a recombinant retroviral vector encoding the firefly luciferase (FLuc) gene was investigated. The resulting Hepa1-6-FLuc cells exhibited similar cellular morphology and biological characteristics, including proliferation, migration and invasion rates, to the parental Hepa1-6 cell line. Furthermore, Hepa1-6-FLuc cells could form tumor masses 
subsequent to their subcutaneous transplantation in nude mice; the bioluminescence signal of the developing tumor masses was continuously enhanced, reflecting cell proliferation and survival in vivo. The Hepa1-6-FLuc cell line, with the stable expression of the FLuc gene, should be an ideal resource to establish hepatocarcinoma animal models, and longitudinally monitor tumor proliferation, viability and metastasis, providing a valuable tool in the study of hepatocarcinoma.

\section{Materials and methods}

Cell culture and chemicals. The murine hepatocellular carcinoma cell line Hepal-6 and human embryonic kidney cell line HEK-293 was purchased from the American Type Culture Collection (Manassas, VA, USA) and maintained in complete Dulbecco's modified Eagle's medium (DMEM) supplemented with $10 \%$ fetal bovine serum (FBS; Hyclone; GE Healthcare Life Sciences, Logan, UT, USA), 100 units/ml penicillin and $100 \mu \mathrm{g} / \mathrm{ml}$ streptomycin at $37^{\circ} \mathrm{C}$ and $5 \% \mathrm{CO}_{2}$. Cells were subcultured at $90 \%$ confluence.

Establishment of Hepal-6 cell lines containing the FLuc gene. A retroviral vector, expressing FLuc and a blasticidin selection marker, and a pCLAmpho mammalian expression vector (Novus Biologicals, LLC, Littleton, CO, USA) were co-transfected into 293 cells with Lipofectamine ${ }^{\circledR} 2000$ (Invitrogen; Thermo Fisher Scientific, Inc., Waltham, MA, USA), according to the manufacture's instructions, to package the recombinant-retrovirus. Hepa1-6 cells were seeded in T-25 flasks and infected with the retrovirus for 7 days, then selected in the presence of $3 \mu \mathrm{g} / \mathrm{ml}$ blasticidin S (Invitrogen; Thermo Fisher Scientific, Inc.) for 14 days. The surviving cells were passaged and designated as Hepa1-6-FLuc.

The in vitro luciferase activity of the Hepa1-6-FLuc cells was assessed by using the Firefly Luciferase Assay kit (Promega Corporation, Madison, WI, USA). A total of $\sim 2 \times 10^{5}$ of cells were incubated in 24-well plates for 3 days and lysed in $1 \mathrm{X}$ passive lysis buffer (PLB). Cell lysate (20 $\mu \mathrm{l})$ and luciferase assay buffer $(100 \mu \mathrm{l})$ were mixed, and the absorbance at $560 \mathrm{~nm}$ was read immediately in the GloMax ${ }^{\circledR}$ 20/20 luminometer (Promega Corporation). The experiment was performed in triplicate.

Cell proliferation and viability assay. An MTT assay and crystal violet staining were used to detect the cell proliferation and viability, as previously described (13). Briefly, $200 \mu 1$ cell suspensions $(\sim 5,000$ cells) were seeded into each well of 96-well plates and incubated overnight. At 1,2,3,4 and 5 days later, $20 \mu \mathrm{l}$ freshly prepared $5 \mathrm{mg} / \mathrm{ml}$ MTT was added to each well. Following a further 4 -h incubation, the medium was carefully removed and $150 \mu \mathrm{l}$ dimethyl sulfoxide was added to dissolve the MTT-formazan crystals. The plate was covered with tinfoil and agitated on an orbital shaker for $15 \mathrm{~min}$, and the absorbance was read at $490 \mathrm{~nm}$.

For crystal violet staining, fixed cells in 24-well plates were stained with $0.05 \%$ crystal violet solution for $30 \mathrm{~min}$ and images were captured using a digital camera at x1 magnification (D7000; Nikon, Tokyo, Japan) after washing three times by PBS. Following treatment with $500 \mu 133 \%$ acetic acid, mission spectra were measured at an excitation wavelength of
$570 \mathrm{~nm}$ using a multimode microplate reader (Thermo Fisher Scientific, Inc.). A total of three independent experiments were performed in duplicate, from which the means and standard deviations (SDs) were calculated.

Colony formation assay. Oncogenic transformation was evaluated with a colony formation assay, as previously described $(14,15)$. A total of 400 cells were seeded onto 6-well plates, and cultured in complete DMEM with $10 \%$ FBS, which was replaced every 3 days. After 14 days, cells were stained with Giemsa stain. The number of the colonies containing $>50$ cells was counted under an inverted phase microscope (TE2000-S; Nikon) at x40 magnification and the plate clone-forming efficiency was calculated as follows: Number of colonies/number of cells seeded x $100 \%$.

Monolayer wound healing cell migration assay. The scratch wound healing assay was performed to detect cell migration in vitro, as previously described (15). Approximately $5 \times 10^{5}$ cells were seeded into 6-well plates in DMEM with $1 \%$ FBS. Following the formation of confluent monolayer, a gap in the surface of the confluent cells was created with a pipette tip. Bright field images at $\times 40$ magnification of the wounds were captured at $0,1,2,3,4$ and 5 days to assess the cell migration across the gap. Each assay was performed in triplicate.

Cell migration and invasion (Matrigel) assay. For the cell migration and invasion assays, a Cell Invasion Assay kit (Cell Biolabs, Inc., San Diego, CA, USA) was used according to the manufacturer's instructions. Briefly, a total of $\sim 1 \times 10^{4}$ cells were seeded into the Transwell insert in serum-free DMEM, whereas DMEM with 10\% FBS was added to the lower well. At $48 \mathrm{~h}$, cells were fixed and cells at the top of chamber were removed. Cells on the lower side of the chamber were stained with crystal violet, and visualized with a light microscope. The stain was dissolved with $33 \%$ acetic acid and absorbance of each well was measured at $570 \mathrm{~nm}$ with a microplate reader (Thermo Fisher Scientific, Inc.). The procedure was repeated independently three times, with triplicate chambers for each group.

Cell implantation and in vivo imaging. The use and care of animals was approved by the Institutional Animal Care and Use Committee of the Children's Hospital of Chongqing Medical University (Chongqing, China). A total of nine female BALB/c nude mice (6-8 weeks, weight $\sim 20 \mathrm{~g}$; Tengxin Biotechnology Co., Ltd, Chongqing, China) were housed in specific pathogen-free laboratory with a $12 / 12 \mathrm{~h}$ light/dark cycle under a controlled temperature of $22 \pm 2{ }^{\circ} \mathrm{C}$ and humidity of $50 \pm 10 \%$ with ad libitum access to food and water. Hepa1-6 cells were infected with adenovirus AdFLuc (Molecular Oncology Laboratory, The University of Chicago Medical Center, Chicago, IL, USA) for $24 \mathrm{~h}$ and termed as Hepa1-6-AdFLuc. Subconfluent Hepa1-6, Hepa1-6-FLuc or Hepa1-6-AdFLuc cells were collected and subcutaneously injected into the front and rear notum on the left and/or right side(s) of the nude mice ( $1 \times 10^{6}$ cells/injection) (16). At 1 day, 1 week and 2 weeks after implantation, mice were intraperitoneally injected with $2 \mathrm{mg} / \mathrm{ml} 0.1 \mathrm{ml}$ D-luciferin (Gold Biotechnology, Inc., Olivette, MO, USA) and visualized using an IVIS-200 optical in vivo 
A
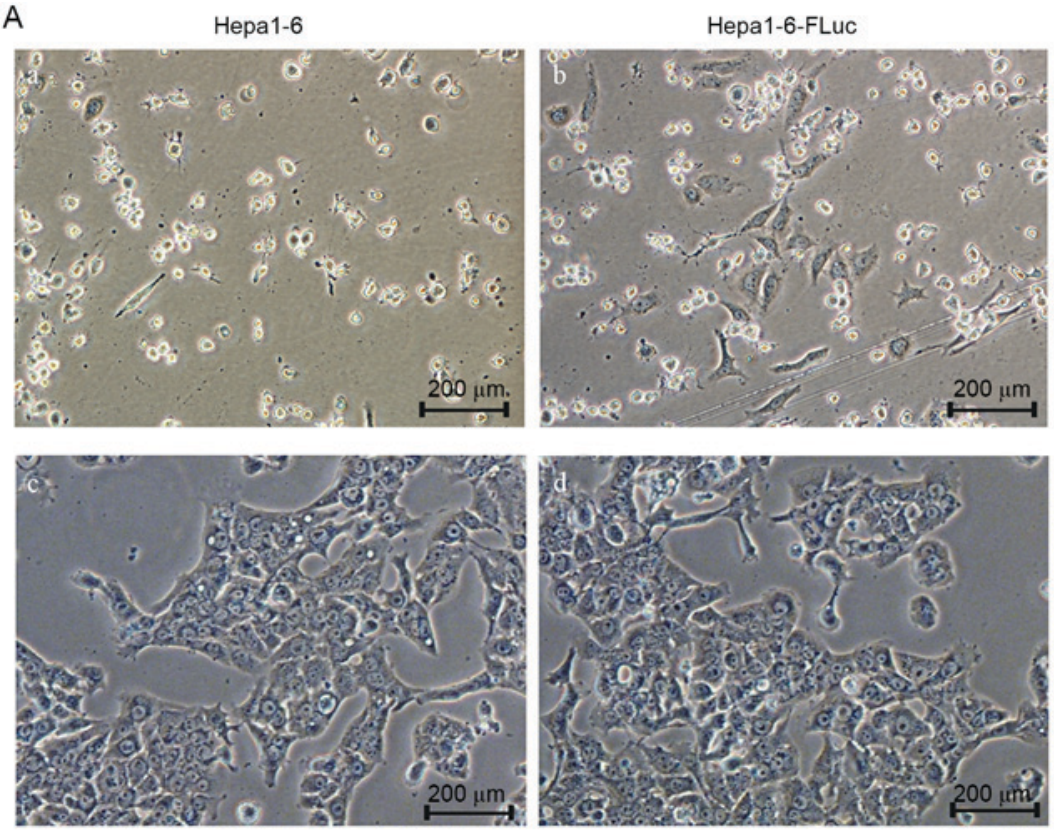

B

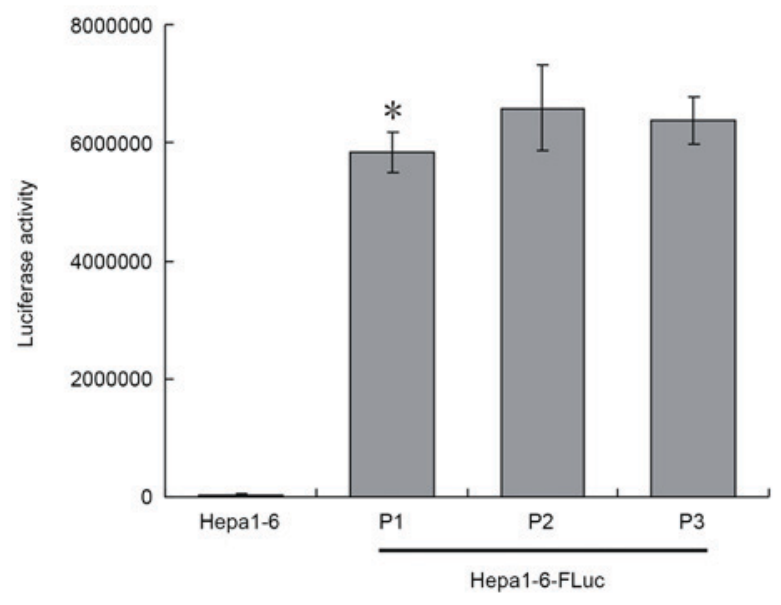

Figure 1. Establishment of the Hepa1-6-FLuc cell line. (A) Blasticidin screening and morphology of Hepa1-6 and Hepa1-6-FLuc cell lines. Hepa1-6 cells were infected with retroviruses expressing the FLuc gene and selected in the presence of $3 \mu \mathrm{g} / \mathrm{ml}$ blasticidin S. (a) Hepa1-6 cells cultured in blasticidin medium for 14 days. (b) Hepa1-6-FLuc cells cultured in blasticidin medium for 14 days. (c) Hepa1-6 cell morphology. (d) Hepa1-6-FLuc cell morphology at passage 5. Magnification x200; Scale bar, $200 \mu \mathrm{m}$. (B) Luciferase activity of Hepa1-6 and Hepa1-6-FLuc cells in 1st, 2nd and 3rd generation subcultures, displayed as relative luminometer units. A total of three independent assays were performed for each group, in triplicate. * P<0.05 vs. Hepa1-6 group. FLuc, firefly luciferase; $\mathrm{P}$, passage generation number.

imaging system (Xenogen Corporation, Alameda, CA, USA) to quantify cell survival.

Assessment of tumor size and histochemical stain. Mice were sacrificed by $\mathrm{CO}_{2}$ asphyxiation at 14 days after cell implantation. Tumor tissues were harvested, the size of the tumors was measured and images were captured using a digital camera. The specimens were fixed with $10 \%$ formalin at room temperature for $30 \mathrm{~min}$, embedded in paraffin and serially cutinto $5-\mu \mathrm{m}$ thick sections. The sections were stained with $1 \%$ hematoxylin and $0.2 \%$ eosin $(\mathrm{H} \& \mathrm{E})$ at room temperature for $10 \mathrm{~min}$, and then photographed with a microscope (Nikon).

Statistical analysis. Data are presented as the means \pm SD and analyzed by SPSS 18.0 software (SPSS, Inc., Chicago,
IL, USA). A two-tailed student's t-test was used to evaluate the difference between two groups, and a one-way analysis of variance with a Student-Newman-Keuls post hoc test was used to evaluate the differences among three or more groups. $\mathrm{P}<0.05$ was considered to indicate a statistically significant difference.

\section{Results}

Establishment of the Hepal-6-FLuc stable cell line. Following 14 days of blasticidin S selection, all Hepa1-6 cells were dead (Fig. 1A-a) and only 5-7\% of Hepa1-6-FLuc cells had survived (Fig. 1A-b). The 5th passage of Hepa1-6-FLuc cells was uniform and exhibited the same cell shape as the Hepa1-6 progenitor cells (Fig. 1A-c and -d). The luciferase activity 
A

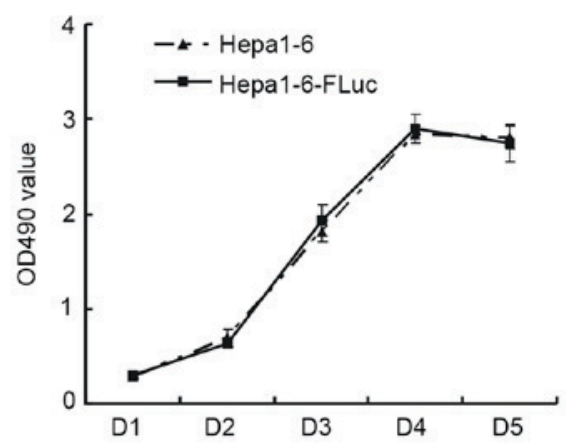

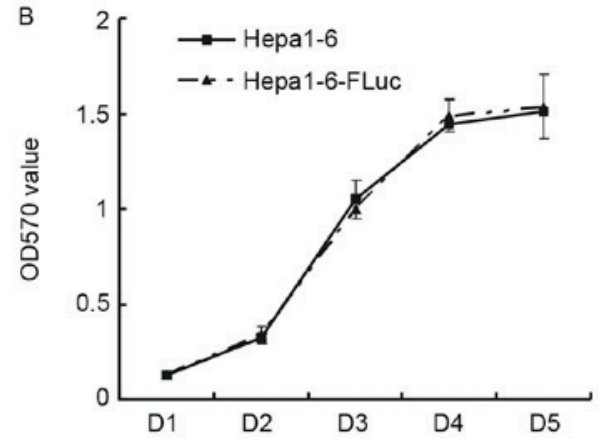

C

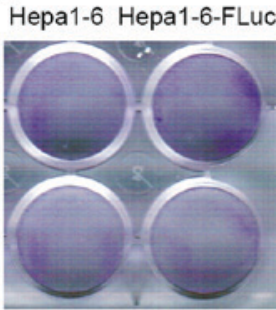

D1

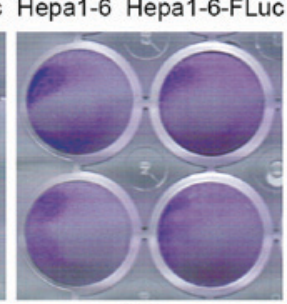

D2

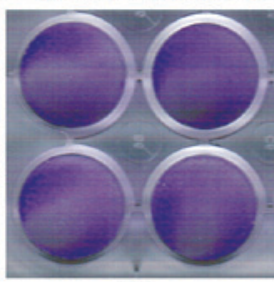

D3

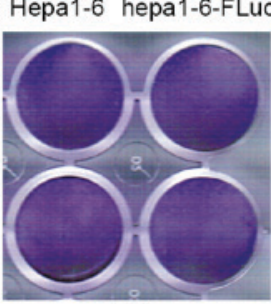

D4

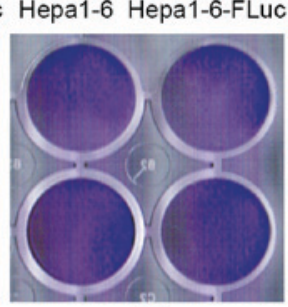

D5

\section{D}
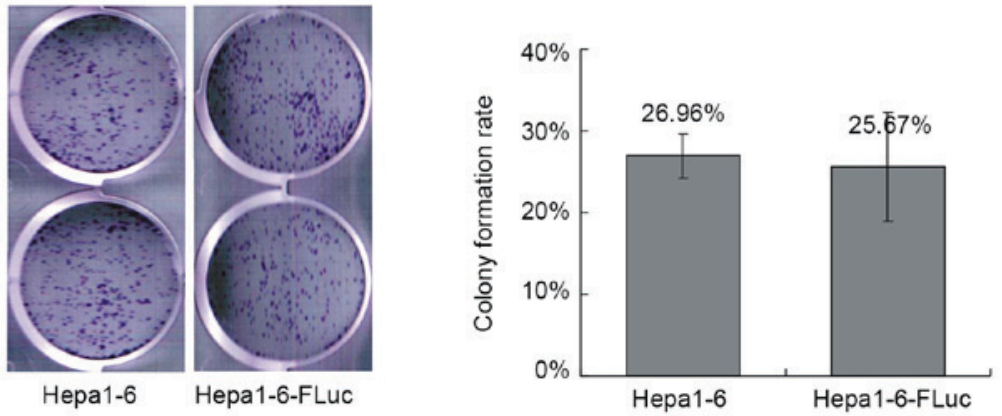

Figure 2. Proliferation and cell viability of Hepa1-6-FLuc and Hepa1-6 cell lines. (A) Cell viability was detected by an MTT assay. (B) Cell proliferation was detected by crystal violet staining. (C) Crystal violet staining of Hepa1-6-FLuc and Hepa1-6 cell lines. Images of stains were captured at day 1, 2, 3, 4 and 5 after seeding. (D) Cell colony formation rate. FLuc, firefly luciferase; OD, optical density; D, day number.

assay revealed significantly increased luciferase activity in the Hepa1-6-FLuc cells compared with the parental Hepa1-6 cells (Fig. 1B; P<0.05). Thus, a stable Hepa1-6-FLuc cell line, with blasticidin resistance and expressing FLuc, was successfully constructed.

Hepa1-6-FLuc cell line has similar characteristics to the Hepal-6 cell line. Growth curves were produced by MTT and crystal violet staining assays. The growth curve of the Hepa1-6 cells exhibited a typical sigmoid shape, and the stable expression of Luc in Hepa1-6-FLuc cells did not affect their proliferation (Fig. 2A-C). Colony formation reflects the proliferation and migration ability of cells; the colony formation rates of Hepa1-6 and Hepa1-6-FLuc cells were 26.59 \pm 2.67 and $25.67 \pm 6.68 \%$, respectively, which were not statistically different (Fig. 2D; P>0.05).

The results of the wound healing assays revealed the migration ability of Hepa1-6 and Hepa1-6-FLuc cells was similar (Fig. 3A). In the Transwell cell migration and invasion assays, the migrating cell number for the Hepa1-6 and Hepa1-6-FLuc groups was consistent (Fig. 3B; P>0.05), which was also true for the invading cell number (Fig. 3C; $\mathrm{P}>0.05$ ). Therefore, the present study demonstrated that the Hepa1-6 and Hepa1-6-FLuc cell lines exhibited similar proliferation, migration and invasion abilities.

Hepa1-6-Fluc cells reflect Hepal-6 cell proliferation and survival in vivo. The Xenogen IVIS imaging system a highly sensitive in vivo imaging system that can be used to track cells in real time if cells are tagged with a gene encoding a luciferase enzyme; its non-invasive visualization allows the monitoring of cell dynamics in vivo $(16,17)$. In the present study, subcutaneous Hepa1-6 tumors were monitored, which exhibited stable FLuc expression or temporary adenovirus-mediated FLuc expression. Hepa1-6, Hepa1-6-FLuc and Hepa1-6-AdFLuc cells were able to form tumor masses. No signal was observed at the Hepa1-6 implantation sites, and the signals of Hepa1-6-FLuc and Hepa1-6-AdFLuc cells were similar at day 1 after implantation (Fig. 4). With increases in tumor size, the luciferase signal of the Hepa1-6-FLuc tumors gradually strengthened, whereas the signal for the Hepa1-6-AdFLuc cells became weaker and eventually disappeared after four weeks. There was no significant difference in tumor sizes observed among the three groups, although the Hepa1-6 AdFLuc tumors were trending towards a larger mean size (Fig. 5A; P>0.05). Based on H\&E staining (Fig. 5B), 


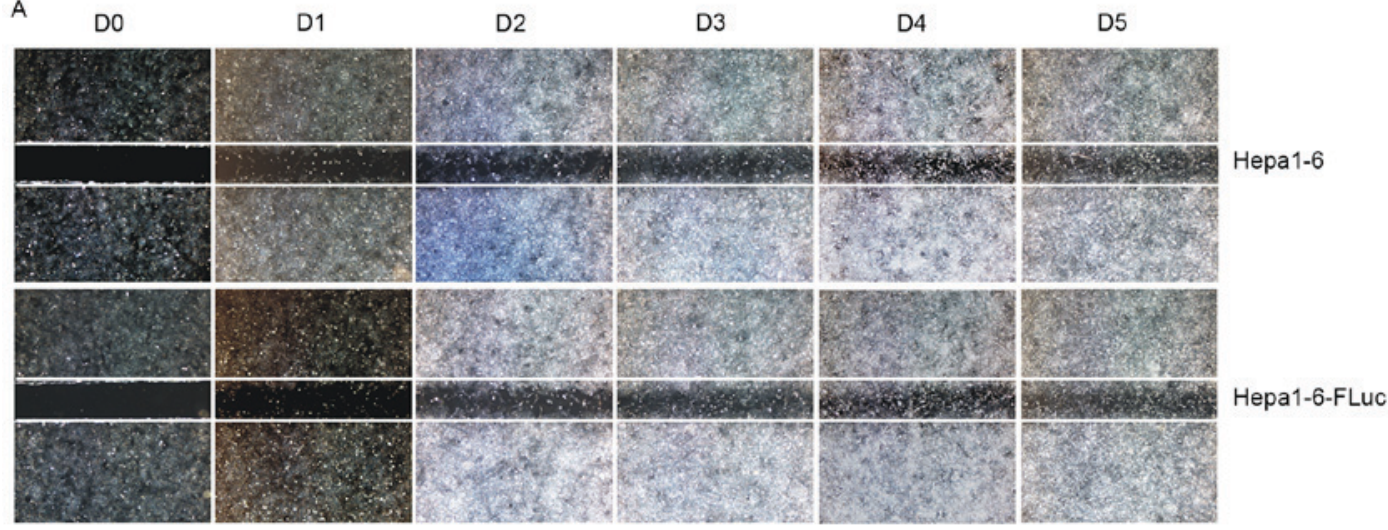

B
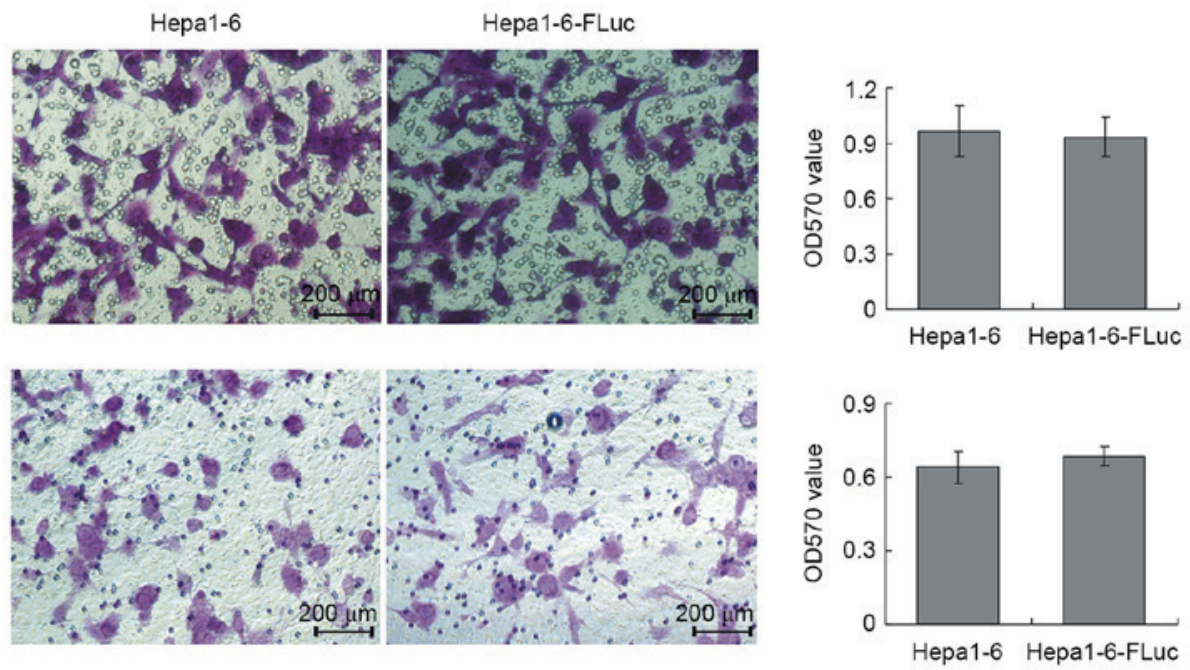

Figure 3. Migration and invasion ability of Hepa1-6-FLuc and Hepa1-6 cell lines. (A) Wound healing assay to detect cell migration ability. Magnification x40. (B) Cell migration as determined by a Transwell assay. Magnification x200; Scale bar, $200 \mu \mathrm{m}$. (C) Cell invasion as determined by a Transwell assay. Magnification x200; Scale bar, $200 \mu \mathrm{m}$. Cells were seeded in Transwell inserts for $48 \mathrm{~h}$ and stained by crystal violet. FLuc, firefly luciferase; OD, optical density; D, day.

it was identified that the Hepa1-6 and Hepa1-6-FLuc cells exhibited polygonal, irregular shapes and different cell sizes, and were arranged densely with deeply stained nuclei, reduced cytoplasm and hyperchromatic mitosis. A number of cells presented with nuclear pyknosis, deep staining or mitotic characteristics. The Hepa1-6-AdFLuc cells formed a tumor mass that exhibited similar cell morphology to the other groups, but with a substantial amount of neovascularization; a number of vessel walls were infiltrated, tumor cells appeared in the vascular lumen and were mixed with blood cells. Therefore, these results suggested that the stable expression of FLuc in Hepa1-6 cells, and not transient adenovirus-mediated expression, did not affect its tumor formation ability in vivo; additionally, this stable expression could be used to monitor cell proliferation and survival in vivo using a cell tracing technique.

\section{Discussion}

The pathogenesis of hepatocellular carcinoma remains incompletely understood at the cellular and molecular levels. The accurate and sensitive evaluation of the effect of therapeutics on in vivo tumor development is also required, as a tool for the longitudinal monitoring of tumor proliferation, viability and metastasis (18-20). Traditional cell tracking methods usually involve histopathological techniques to observe the labeled cells in vivo, which require the isolation of samples from patients or animals at different time-points. Although this method allows the collection of data from the transplanted cell tumors at the time of sacrifice, it does not allow the collection of real-time dynamic information of cell location, viability, migration, activation and differentiation in vivo $(21,22)$. BLI is a novel non-invasive technique for obtaining biomedical images of living tissues at the cellular and molecular levels, which may be utilized to constantly monitor the physiological, biochemical and pathological processes of diseases in vivo. Compared with other techniques, BLI is preferable because of its non-invasion, high sensitivity and dynamic monitoring (23-25), and serves an important role in quantitatively assessing in vivo tumor cell proliferation and invasion over time. In the study of pre-clinical oncology, BLI is a versatile and sensitive tool that is based on the detection of light emission from cells or tissues. Live animal imaging of small animal tumor models using BLI involves the production of light by luciferase-expressing cells in the animal in the presence of substrate $(24,26-28)$. 


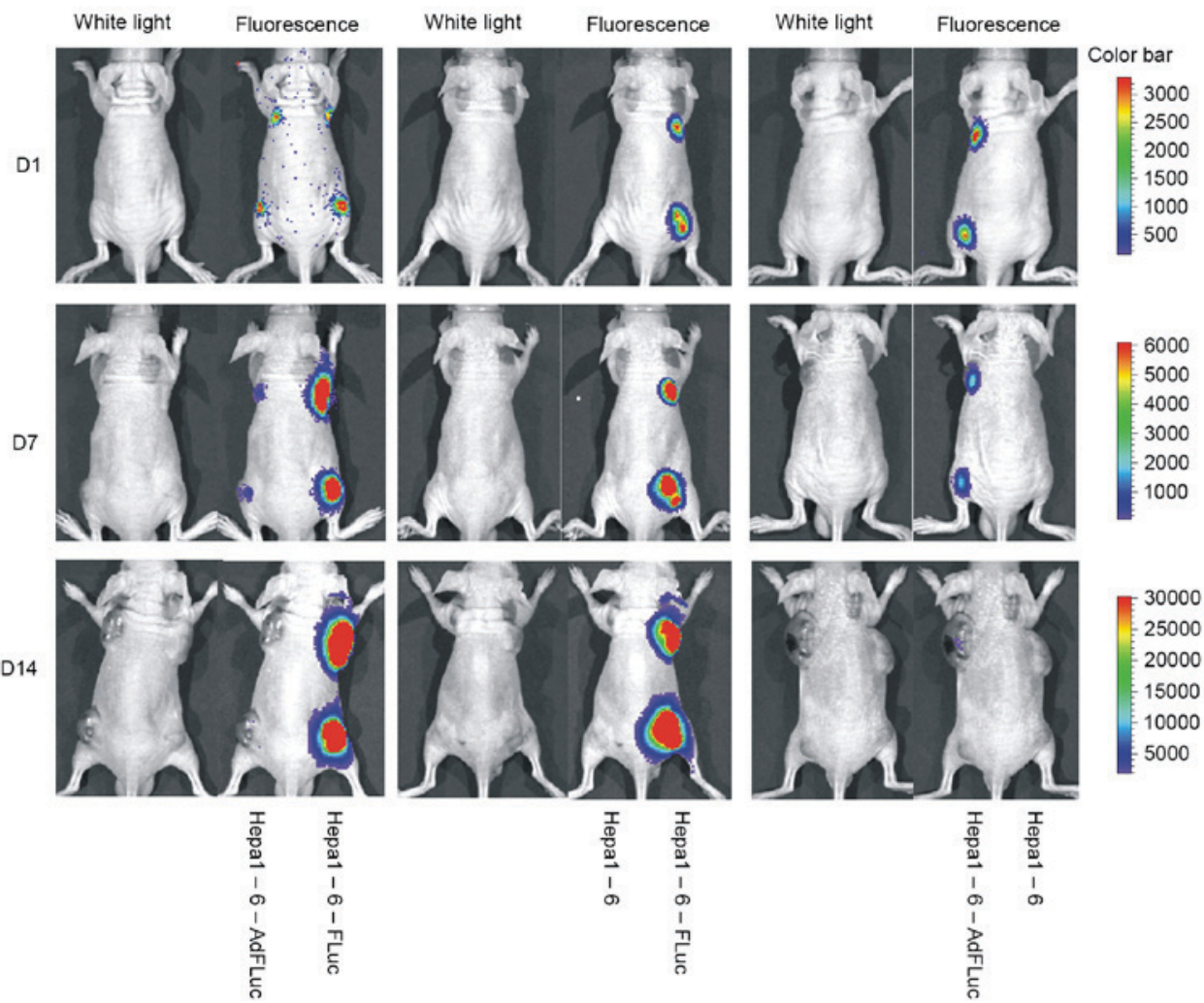

Figure 4. Bioluminescence imaging of Hepa1-6-FLuc, Hepa1-6-AdFLuc and Hepa1-6 cell tumors. Hepa1-6, Hepa1-6-FLuc, and Hepa1-6-AdFLuc cells were subcutaneously injected into nude mice. White light and fluorescence images in the same field of view were captured at 1,7 and 14 days subsequent to implantation and D-Luciferin injection. FLuc, firefly luciferase integrated by retrovirus; AdFLuc, firefly luciferase from adenovirus.
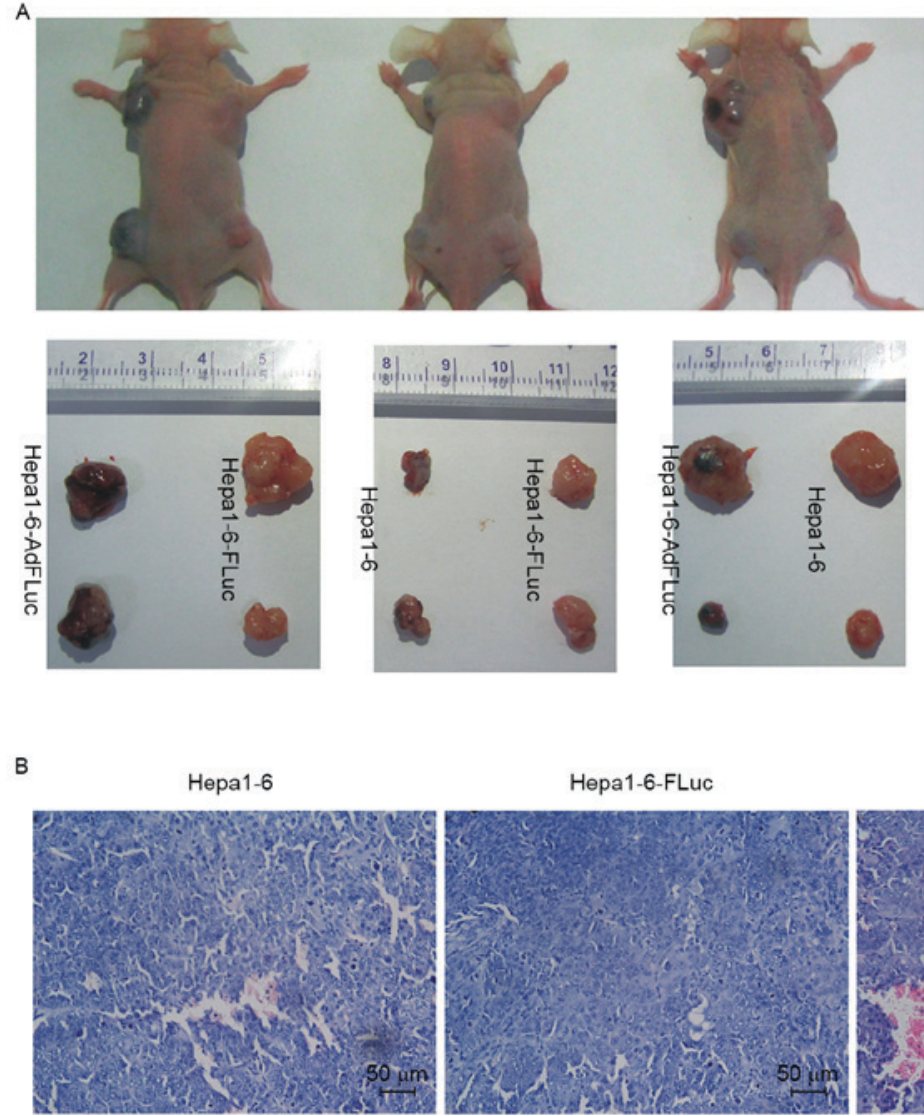

Hepa1-6-FLuc
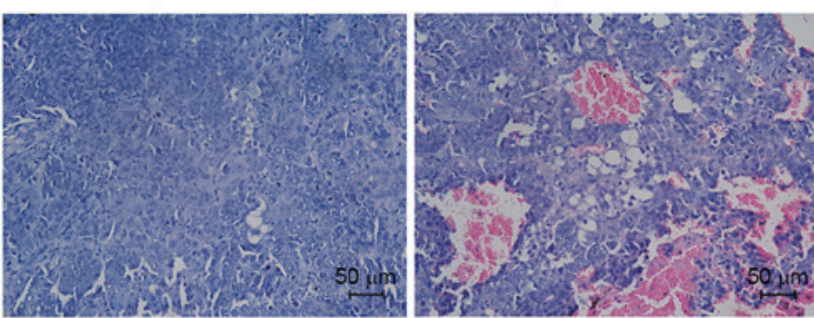

Figure 5. Gross specimens and histological sections of tumor masses derived from Hepa1-6-FLuc and Hepa1-6 cell tumors. (A) Mice were sacrificed after 14 days of cell implantation. The diameter of tumor mass was measured. (B) Hematoxylin and eosin staining of the tumor masses. Scale bar, $50 \mu \mathrm{m}$. FLuc, firefly luciferase integrated by retrovirus; AdFLuc, firefly luciferase from adenovirus.

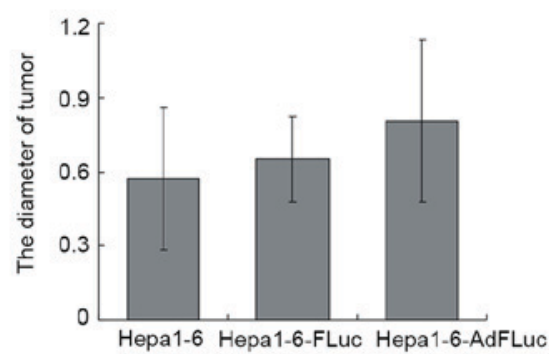

Hepa1-6-AdFLuc 
The FLuc gene has been isolated from the cDNA library of Photinus pyralis. Luciferases emit light in the presence of D-luciferin substrate, ATP, magnesium and oxygen, which is a valuable tool for noninvasively monitoring cells in vivo $(24,29)$. A number of studies (30-32) have demonstrated the use of recombinant adenoviruses as a gene delivery vector to express the FLuc gene in different animal cells. However, in the present study it was identified that adenovirus medicated FLuc expression was not long-lasting due to its low and transient level of transgenic expression, potentially as a result of cellular immunity. Most importantly, in a successful hepatocarcinoma model, the transplanted cells should proliferate in vivo and gradually form a mass, but the signals of the AdFLuc-labeled cells in the present study were not consistent with the growth of the tumors. Adenoviruses do not integrate the reporter gene into the host cell genome, preventing the tracing of the daughter cells originating from the transplanted tumor cells in vivo $(33,34)$.

In the present study, a Hepa1-6-FLuc cell line with FLuc gene expression was constructed by retroviral infection. The passaged Hepa1-6-FLuc cells stably expressed FLuc activity, and exhibited similar morphology, proliferation, migration and invasion characteristics compared with the Hepa1-6 cells. The FLuc gene was replicated with Hepa1-6-FLuc cell proliferation in vivo following the implantation in nude mice, therefore luciferin-mediated BLI traced the implanted cell accurately. No difference in tumor mass volume between the Hepa1-6-FLuc and Hepa1-6 cell masses was observed, but the volume of the AdFLuc-infected Hepa1-6 cell tumor mass was non-significantly increased compared with that of the Hepa1-6 cell tumors. In addition, hemorrhage and blood cells were present in the gross specimens and histological sections of Hepa1-6 AdFLuc tumors, indicating that adenovirus infection may promote the neovascularization and development of Hepa1-6 tumors (35). Therefore, compared with adenovirus-based methods, the retrovirus-mediated stable expression of exogenous FLuc gene may more accurately label and trace cell survival and proliferation in vivo.

In conclusion, the present study describes a hepatocarcinoma cell line that stably expressed the FLuc gene. The Hepa1-6-FLuc cells exhibited the same cellular characteristics as the Hepa1-6 progenitor cells, were able to replace Hepa1-6 cells in the establishment of a hepatocarcinoma animal model, and may be useful for the future study of tumor pathogenesis and the screening of novel anticancer drugs for the treatment of hepatocarcinoma.

\section{Acknowledgements}

Not applicable.

\section{Funding}

The present study was supported by a research grant from the Natural Science Foundation of Chongqing City (grant no. csct2016jcyjA0228) and the National Natural Science Foundation of China (grant no. 81100309).

\section{Availability of data and materials}

All data generated or analyzed during this study are included in this published article.

\section{Authors' contributions}

YL carried out the animal experiments. MNL and JJC carried out the cell experiments. KY and LZ did pathological histochemistry and helped to evaluate bioluminescence imaging. YW and MJG participated in cell culture. YH executed statistical analyses. TCH and YB designed the research and wrote the manuscript. All authors read and approved the final manuscript.

\section{Ethics approval and consent to participate}

The use and care of animals within the study was approved by the Institutional Animal Care and Use Committee of the Children's Hospital of Chongqing Medical University (Chongqing, China).

\section{Consent for publication}

Not applicable.

\section{Competing interests}

The authors declare that they have no competing interests.

\section{References}

1. Wang CH, Wey KC, Mo LR, Chang KK, Lin RC and Kuo JJ: Current trends and recent advances in diagnosis, therapy, and prevention of hepatocellular carcinoma. Asian Pac J Cancer Prev 16: 3595-3604, 2015.

2. Wallace MC, Preen D, Jeffrey GP and Adams LA: The evolving epidemiology of hepatocellular carcinoma: A global perspective. Expert Rev Gastroenterol Hepatol 9: 765-779, 2015.

3. Zhu RX, Seto WK, Lai CL and Yuen MF: Epidemiology of hepatocellular carcinomain the Asia-pacific region. Gut Liver 10: 332-339, 2016.

4. Zhu Q, Li N, Zeng X, Han Q, Li F, Yang C, Lv Y, Zhou Z and Liu Z: Hepatocellular carcinoma in a large medical center of China over a 10 -year period: Evolving therapeutic option and improving survival. Oncotarget 6: 4440-4450, 2015

5. Zhao GJ, Xu LX, Chu ES, Zhang N, Shen JY, Damirin A and Li XX: Establishment of an orthotopic transplantation tumor model of hepatocellular carcinoma in mice. World $\mathrm{J}$ Gastroenterol 18: 7087-7092, 2012.

6. Heindryckx F, Colle I and Van Vlierberghe H: Experimental mouse models for hepatocellular carcinoma research. Int J Exp Pathol 90: 367-386, 2009.

7. Kircher MF, Gambhir SS and Grimm J: Noninvasive cell-tracking methods. Nat Rev Clin Oncol 8: 677-688, 2011

8. Masuda H, Okano HJ, Maruyama T, Yoshimura Y, Okano H and Matsuzaki Y: In vivo imaging in humanized mice. Curr Top Microbiol Immunol 324: 179-196, 2008.

9. Kim JE, Kalimuthu S and Ahn BC: In vivo cell tracking with bioluminescence imaging. Nucl Med Mol Imaging 49: 3-10, 2015.

10. Huang NF, Okogbaa J, Babakhanyan A and Cooke JP: Bioluminescence imaging of stem cell-based therapeutics for vascular regeneration. Theranostics 2: 346-354, 2012.

11. Madero-Visbal RA, Colon JF, Hernandez IC, Limaye A, Smith J, Lee CM, Arlen PA, Herrera L and Baker CH: Bioluminescence imaging correlates with tumor progression in an orthotopic mouse model of lung cancer. Surg Oncol 21: 23-29, 2012.

12. Wang Q, Luan W, Goz V, Burakoff SJ and Hiotis SP: Non-invasive in vivo imaging for liver tumour progression using an orthotopic hepatocellular carcinoma model in immunocompetent mice. Liver Int 31: 1200-1208, 2011.

13. He Y, Zhou JW, Xu L, Gong MJ, He TC and Bi Y: Comparison of proliferation and differentiation potential between mouse primary hepatocytes and embryonic hepatic progenitor cells in vitro. Int J Mol Med 32: 476-484, 2013. 
14. Xu J, Yong M, Li J, Dong X, Yu T, Fu X and Hu L: High level of CFTR expression is associated with tumor aggression and knockdown of CFTR suppresses proliferation of ovarian cancer in vitro and in vivo. Oncol Rep 33: 2227-2234, 2015.

15. Cui J, Gong M, He Y, Li Q, He T and Bi Y: All-trans retinoic acid inhibits proliferation, migration, invasion and induces differentiation of hepa1- 6 cells through reversing EMT in vitro Int J Oncol 48: 349-357, 2016.

16. Bi Y, He Y, Huang J, Su Y, Zhu GH, Wang Y, Qiao M, Zhang BQ, Zhang $\mathrm{H}$, Wang $\mathrm{Z}$, et al: Functional characteristics of reversibly immortalized hepatic progenitor cells derived from mouse embryonic liver. Cell Physiol Biochem 34: 1318-1338, 2014.

17. Henriques C, Henriques-Pons A, Meuser-Batista M, Ribeiro AS and de Souza W: In vivo imaging of mice infected with bioluminescent Trypanosoma cruzi unveils novel sites of infection. Parasit Vector 7: 89, 2014.

18. Chen X, Yin S, Hu C, Chen X, Jiang K, Ye S, Feng X, Fan S, $\mathrm{Xie} \mathrm{H}$, Zhou L and Zheng S: Comparative study of nanosecond electric fields in vitro and in vivo on hepatocellular carcinoma indicate macrophage infiltration contribute to tumor ablation in vivo. PLoS One 9: e86421, 2014.

19. Hossain MA, Kim DH, Jang JY, Kang YJ, Yoon JH, Moon JO, Chung HY, Kim GY, Choi YH, Copple BL and Kim ND: Aspirin enhances doxorubicin-induced apoptosis and reduces tumor growth in human hepatocellular carcinoma cells in vitro and in vivo. Int J Oncol 40: 1636-1642, 2012.

20. Kwak MS, Yu SJ, Yoon JH, Lee SH, Lee SM, Lee JH, Kim YJ, Lee HS and Kim CY: Synergistic anti-tumor efficacy of doxorubicin and flavopiridol in an in vivo hepatocellular carcinoma model. J Cancer Res Clin Oncol 141: 2037-2345, 2015.

21. Gu E, Chen WY, Gu J, Burridge P and Wu JC: Molecular imaging of stem cells: Tracking survival, biodistribution, tumorigenicity, and immunogenicity. Theranostics 2: 335-345, 2012.

22. Alam F and Yadav N: Potential applications of quantum dots in mapping sentinel lymph node and detection of micrometastases in breast carcinoma. J Breast Cancer 16: 1-11, 2013.

23. Heffern MC, Park HM, Au-Yeung HY, Van de Bittner GC, Ackerman CM, Stahl A and Chang CJ: In vivo bioluminescence imaging reveals copper deficiency in a murine model of nonalcoholic fatty liver disease. Proc Natl Acad Sci USA 113: 14219-14224, 2016.

24. Sun A, Hou L, Prugpichailers T, Dunkel J, Kalani MA, Chen X, Kalani MY and Tse V: Firefly luciferase-based dynamic bioluminescence imaging: A noninvasive technique to assess tumor angiogenesis. Neurosurgery 66: 751-757, 2010.

25. Wang R, Zhang K, Tao H, Du W, Wang D, Huang Z, Zhou M, $\mathrm{Xu}$ Y, Wang Y, Liu N, et al: Molecular imaging of tumor angiogenesis and therapeutic effects with dual bioluminescence. Curr Pharm Biotechnol 18: 422-428, 2017.
26. Brutkiewicz S, Mendonca M, Stantz K, Comerford K, Bigsby R, Hutchins G, Goebl M and Harrington M: The expression level of luciferase within tumour cells can alter tumour growth upon in vivo bioluminescence imaging. Luminescence 22: 221-228, 2007.

27. Mezzanotte L, Fazzina R, Michelini E, Tonelli R, Pession A, Branchini B and Roda A: In vivo bioluminescence imaging of murine xenograft cancer models with a red-shifted thermostable luciferase. Mol Imaging Biol 12: 406-414, 2010.

28. Hemmati R, Hosseinkhani S, Sajedi RH, Azad T, Tashakor A, Bakhtiari $\mathrm{N}$ and Ataei F: Luciferin-regenerating enzyme mediates firefly luciferase activation through direct effects of D-cysteine on luciferase structure and activity. Photochem Photobiol 91: 828-836, 2015.

29. Wu JC, Sundaresan G, Iyer M and Gambhir SS: Noninvasive optical imaging of firefly luciferase reporter gene expression in skeletal muscles of living mice. Mol Ther 4: 297-306, 2001.

30. Wang F, Wang Z, Tian H, Qi M, Zhai Z, Li S, Li R, Zhang H, Wang W, Fu S, et al: Biodistribution and safety assessment of bladder cancer specific recombinant oncolytic adenovirus in subcutaneous xenografts tumor model in nude mice. Curr Gene Ther 12: 67-76, 2012.

31. Cao L, Zeng Q, Xu C, Shi S, Zhang Z and Sun X: Enhanced antitumor response mediated by the codelivery of paclitaxel and adenoviral vector expressing IL-12. Mol Pharm 10: 1804-1814, 2013.

32. Man K, Ng KT, Xu A, Cheng Q, Lo CM, Xiao JW, Sun BS, Lim ZX, Cheung JS, Wu EX, et al: Suppression of liver tumor growth and metastasis by adiponectin in nude mice through inhibition of tumor angiogenesis and downregulation of Rho kinase/IFN-inducible protein 10/matrix metalloproteinase 9 signaling. Clin Cancer Res 16: 967-977, 2010.

33. Volpers C and Kochanek S: Adenoviral vectors for gene transfer and therapy. J Gene Med 6 (Suppl 1): S164-S171, 2004.

34. Hall K, Blair Zajdel ME and Blair GE: Unity and diversity in the human adenoviruses: Exploiting alternative entry pathways for gene therapy. Biochem J 431: 321-336, 2010.

35. Suzuki K, Sun R, Origuchi M, Kanehira M, Takahata T, Itoh J, Umezawa A, Kijima H, Fukuda S and Saijo Y: Mesenchymal stromal cells promote tumor growth through the enhancement of neovascularization. Mol Med 17: 579-587, 2011.

This work is licensed under a Creative Commons Attribution-NonCommercial-NoDerivatives 4.0 International (CC BY-NC-ND 4.0) License. 